ALEXANDER HÖLLWERTH

\title{
Schuldverstrickung und Identitätssuche angesichts der Shoah bei Robert Schindel, Doron Rabinovici und Martin Pollack
}

Artykuł omawia trzy powieści: Roberta Schindela Gebürtig (1992), Dorona Rabinovici Suche nach M. (1997) i Martina Pollacka Der Tote im Bunker (2004), których autorzy podejmują obecną w międzypokoleniowych relacjach problematykę zakłamywania winy i poszukiwania tożsamości. Należą oni do tzw. ,drugiego pokolenia ‘ po Shoah. Robert Schindel (rocznik 1944) und Doron Rabinovici (rocznik 1961) są potomkami ofiar Shoah, Martin Pollack (rocznik 1944) jest synem nazistowskiego zbrodniarza. Zajmują oni poczesne miejsce pośród tych austriackich pisarzy, którzy w swej twórczości m. in. podejmują próbę uporania się z problemem udziału Austriaków w ludobójstwie Żydów.

Um Schuldverstrickungen und Identitätssuche zwischen den Generationen geht es in allen drei Texten, auf die im vorliegenden Artikel genauer eingegangen wird: Robert Schindels Gebürtig (1992), Doron Rabinovicis Suche nach M. (1997) und Martin Pollacks Der Tote im Bunker (2004). Alle drei Autoren gehören der sog. ,zweiten Generation" nach der Shoah an: Robert Schindel (geb. 1944) und Doron Rabinovici (geb. 1961) sind Nachgeborene von Opfern der Shoah, Martin Pollack (geb. 1944) ist der Sohn eines NS-Verbrechers. Alle drei Autoren positionieren sich innerhalb der österreichischen Literatur nicht zuletzt dadurch, dass sie einen Beitrag zur Aufarbeitung der österreichischen Beteiligung am Verbrechen der Judenvernichtung zu leisten versuchen.

The article discusses three Austrian novels: Robert Schindel's Gebürtig (1992), Doron Rabinovici's Suche nach M. (1997) and Martin Pollack's Der Tote im Bunker (2004) whose authors undertake to analyse guilt entanglement and identity quest in selfimages of different generations. The authors belong to the so-called , second generation' after Shoah. Robert Schindel (b. 1944) und Doron Rabinovici (b. 1961) are children of the victims of Shoah; Martin Pollack (b. 1944) is a Nazi murderer's son. They are among prominent Austrian writers who are not indifferent to the issue of the Austrians' responsibility for the genocide of Jews. 


\section{Intergenerationelles Schuldtrauma von Ödipus zu Dibbuk}

Das ,kommunikative Gedächtnis“ umfasst nach dem Ägyptologen JAN AssMANN (2005:50) einen Erinnerungsraum, der den drei bis vier Generationen entspreche, die in der Bibel für eine Schuld einstehen müssen. Ein solches Verständnis einer Schuld, die intergenerationell ,weitergegeben“ wird, liegt auf einer anderen Ebene als jene „Aufmerksamkeit auf die Person des Einzelnen“ (ARENDT 2008:21), in der Hannah Arendt die große Bedeutung der Rechtsprechung in Bezug auf Nazi-Verbrecher wie etwa Adolf Eichmann u. a. sieht. Alle nicht spezifischen, abstrakten Rechtfertigungen, angefangen vom ,Zeitgeist' bis zum Ödipuskomplex, würden vor Gericht zusammenbrechen (ARENDT 2008:21). Diese Aussage Arendts kann jedoch nicht dahingehend verstanden werden, dass ,Zeitgeist" und Ödipuskomplex in einem rechtsstaatlichen Gerichtsprozess keine Rolle spielen können - vielmehr kann Arendt nur meinen: Vor Gericht zu verantworten haben sich nicht ,Zeitgeist ${ }^{\text {* }}$ und Ödipuskomplex, sondern ein Einzelner. Auf einer etwas anderen Ebene stellt dies Arendt klar: Die Frage laute nicht, wie das System funktioniert habe, sondern vielmehr, warum der Angeklagte ein Funktionär in diesem System geworden sei (ARENDT 2008:22). Um auf diese Frage eine Antwort zu finden, können nun sehr wohl wieder ,Zeitgeist ' und Ödipuskomplex ins Spiel gebracht werden, nicht jedoch so sehr als exkulpierende, sondern als explizierende Momente.

Der Ödipuskomplex verweist auf die Gestalt der griechischen Tragödie, den König Ödipus, der durch seine bloße Geburt schon in einen Schuldzusammenhang verstrickt ist. Das Geschehen des König Ödipus kann als Weg vom Schein zum Sein, das an den Tag und zur Wahrheit Kommen des Verborgenen betrachtet werden. Wahrheit und Licht seien in der griechischen Sprache identisch: a-letheia, der Zustand des Entborgenseins, des aus der Verhüllung Heraustretens (STEINMANN 2002:79). ,In die Wahrheit treten“ im Sinne der Sophokles'schen Tragödie bedeutet, die eigene unschuldige Verstrickung in einen intergenerationellen Schuldzusammenhang zu erkennen.

,Unschuldig schuldig' werden auch die Protagonisten in der dramatischen Legende Dibbuk des ostjüdischen Dichters An-Ski (eigentlich Schlomo S. Rappoport, 1863-1920), die 1920 in Warschau uraufgeführt wurde: Zwei Talmudstudenten, Sender und Nissan, geloben einander, später einmal ihre Kinder zu vermählen, falls der eine eine Tochter und der andere einen Sohn haben würde. Nissan stirbt in der Fremde. Viele Jahre später genießt sein Sohn Chanan als fremder Talmudstudent bei Sender Freitisch. Senders Toch- 
ter Lea und Chanan verlieben sich ineinander. Sender jedoch, der nicht weiß, dass Chanan Nissans Sohn ist, verlobt seine Tochter mit einem reicheren Bewerber. Als Chanan dies erfährt, stirbt er, und sein Geist fährt im Augenblick der Vermählung Leas mit seinem reicheren Konkurrenten als Dibbuk ${ }^{1}$ in deren Leib. Sender, der das seinem ehemaligen Freund Nissan gegebene Versprechen bereits vergessen (bzw. verdrängt) hat, wird nun von diesem vor das Thoragericht zitiert, wo sein Wortbruch an den Tag kommt. Ihm werden Bußgebete und hohe Geldspenden an die Armen auferlegt. Beim darauffolgenden Exorzismus wird der Dibbuk aus Leas Körper vertrieben, worauf auch diese stirbt.

Wenn zwar die kulturellen Kontexte, in denen Sophokles' König Ödipus und An-Skis Dibbuk jeweils stehen, andere sind, so ist doch das Grundschema ein ähnliches: Die Kinder sind in einen Schuldzusammenhang verstrickt, den die Väter ,gestiftet' (bzw. ,angestiftet') haben. Dieser Zusammenhang ist den Kindern anfänglich nicht bewusst (unbewusst) und wird ihnen gerade dadurch zum Verhängnis. Während im König Ödipus die Tragödie erst dadurch in Gang kommt, dass seine Eltern dem ihnen prophezeiten (unentrinnbaren) Schicksal entgehen wollen, kommt es in An-Skis Dibbuk zur Tragödie dadurch, dass Reb Sender sich nicht erinnern will. Und nicht kommunizieren will. Im Dibbuk fragt der tote Nissan seinen Freund Sender über Reb Schimschon: „Nissan, Sohn der Rebbeka, fragt, warum du seinen Sohn, als er dein Haus betrat und an deinem Tisch saß, kein einziges Mal gefragt hast, wer er ist und woher er stammt?" (AN-SKI 1976:45) Sender gibt zu, dass er es versäumt habe, ihn zu fragen. Darauf antwortet Nissan, Sender habe seinen Sohn tief im Herzen erkannt und deshalb nicht fragen wollen, worauf dieser sein Gesicht bedeckt und lautlos weint (AN-SKI 1976:45f.). Die Schuld von Sender hat drei Schichten: Auf der ersten Schicht liegt die objektive Schuld (das nichteingehaltene Versprechen) - diese Schuld ließe sich durch Nicht-Wissen und durch Vergessen relativieren. Auf der zweiten Schicht wird aber sichtbar, dass dem Vergessen eine Intention zugrunde lag - er wollte für seine Tochter ein bequemes Leben an der Seite eines reichen Mannes haben. Auf der dritten Schicht kommt die eigentliche Schuld von Sender zutage. Er hat im Tiefsten gewusst, wer der fremde Talmudschüler ist und hat seinen ,Gedächtnis-

Ein ,Dibbuk' [zu finden unter: díbek (m.), dibukim (Pl.)] wird in der JiddischEnzyklopädie von Leo Rosten (2006:161) definiert als: 1.) Böser Geist, in der Regel die Seele eines Toten, die sich bei einem Lebenden einnistet, auf den der Tote eines Anspruch hatte 2.) Ein Dämon, der von jemandem Besitz ergreift und ihn verrückt, irrational, sündig, korrupt und verbrecherisch macht. 
Alexander Höllwerth

verlust ‘ nur vorgeschützt. Durch Kommunikation, die er bewusst-unbewusst verweigert hat, hätte er seinem Gedächtnis auf die Sprünge helfen können. In diesem Zusammenhang gewinnt der Begriff des ,kommunikativen Gedächtnisses' eine tiefenpsychologische Dimension: Der Akt einer bewusst-unbewussten Verdrängung ${ }^{2}$ blockiert das ,kommunikative Gedächtnis‘. Die durch diese Kommunikations- und Erinnerungsverweigerung intendierte Entlastung der folgenden Generationen von der Schuld der Väter (Sender will ein bequemes Leben für Lea) verstrickt diese noch mehr in deren Schuld.

Durch die Erfahrung der Shoah bekam die Frage der Schuld eine bislang ungeahnte Dimension, auf die vorerst, wie Arendt es formulierte, sprachloses Entsetzen eine adäquate Reaktion ist. Gegen dieses sprachlose Entsetzen bringt Arendt die rechtliche Dimension ins Spiel: Im Gerichtssaal löse sich dieses auf. Man hätte es mit Personen in dem geordneten Diskurs von Anklage, Verteidigung und Urteilsspruch zu tun (ARENDT 2008:22). ${ }^{3}$ Doch vermögen auch Gerichtsprozesse wie der Eichmann-Prozess in Jerusalem im Jahre 1961 oder der Auschwitz-Prozess, der in Frankfurt am Main von 1963 bis 1965 stattfand, die durch die Shoah aktualisierte Dimension der Schuld nicht zu erschöpfen, weil das Problem, wie es der italienische Philosoph GIORGIO AGAMBEN (2003:17) ausdrückt, so enorm war, „daß es das Recht selbst in Frage stellte und mit sich in den Abgrund riß“. Das Netz der Schuldverstrickung nach der Shoah ist, metaphorisch gesprochen, weiter gespannt, als dass es mit Hilfe eines rechtsstaatlichen Gerichtsprozesses aufgelöst werden könnte. In dieses Netz sind nicht nur die unmittelbaren Täter, die man vor Gericht stellen kann, sondern auch, paradoxerweise, deren Opfer wie deren Kinder und Enkelkinder ebenso wie die Kinder und Enkelkinder der Täter verstrickt, die, um es mit ROBERT SCHINDEL (1992:244) zu formulieren, „vollkommen unschuldig“ zu ihrer Schuld gekommen sind. Der über die Zeitzeugen sowohl auf Täter- als auch auf Opferseite hinausgehenden intergenerationellen Schuldverstrickung ist jedoch juristisch nicht mehr beizukommen: Die Betroffenen können für das Verbrechen weder im juristi-

2 Die Verdrängung definiert Freud (1989:291f.) in der 19. Vorlesung zur Einführung in die Psychoanalyse als Sträuben gegen das Bemühen, das Unbewusste ins Bewusste zu überführen.

3 Ähnlich argumentiert auch der Philosoph KARL JASPERS (1963:50) angesichts der durch die Verbrechen des nationalsozialistischen Regimes akut gewordenen Schuldfrage im Jahre 1946: „Sobald der Rechtsgedanke auftaucht, kann verhandelt werden, um das wahre Recht durch Diskussion und methodische Verfahren zu finden." 
schen noch im moralischen Sinne eine unmittelbare Verantwortung übernehmen. Es sind vielmehr psychoanalytische (sozialpsychologische) Verfahren, die dabei helfen, einen Umgang mit dem ,entlehnten unbewussten Schuldgefühl“" (FREUD 2000:317) bei den Nachkommen der Opfer ebenso wie der Täter zu finden, indem sie das Unbewusste bewusstzumachen, das NichtKommunizierte zu verbalisieren trachten. Allerdings gilt es bei allen Analogien, die in psychotraumatologischen Studien zwischen der Übertragung von Traumata in Opfer- und Täterfamilien konstatiert werden, die Differenz zwischen der Weitergabe eines Schuldgefühls in Opferfamilien und der in Täterfamilien hervorzuheben: Während sich das ,entlehnte Schuldgefühl' der Kinder von Nazi-Tätern auf eine reale Schuld von deren Eltern im juristischen bzw. moralischen Sinn bezieht, bezieht sich das Schuldgefühl der Opferkinder auf das Überleben selbst, das als Schuld gegenüber denen gedeutet wird, die nicht überlebt haben (RoTHE 2009:16-19). Für dieses Schuldgefühl fand der Auschwitzüberlebende JEAN AMÉRY (1988:106) eine radikale Formulierung: „Jude sein, das hieß für mich von diesem Anfang an, ein Toter auf Urlaub sein, ein zu Ermordender, der nur durch Zufall noch nicht dort war, wohin er rechtens gehörte, und dabei ist es in vielen Varianten, in manchen Intensitätsgraden bis heute geblieben." Diese Empfindung, „ein Toter auf Urlaub zu sein“ (d.h. eine Ausnahme von der Regel), der sein Überleben einem ,unrechtmäßigen' Zufall verdankt, wird an die Kinder der Überlebenden weitergegeben. Doron Rabinovici formuliert das ambivalente Lebensgefühl der ,zweiten Generation “ nach der Shoah wie folgt:

Vor ihrer Geburt war der Tod, der Massenmord. Ihr Leben ist eine Ausnahme, gründet im Zufall und Glück des Überlebens ihrer Eltern. Sie sind ein Widerspruch, der dem Verbrechen und seinen Zielen, der deutschen Vergangenheit und Gegenwart entgegenhallt. (Zit. nach TeufEL / SchMitz 2007:215)

\section{Die Autoren der ,zweiten Generation‘ - Erzählen als Katharsis}

Ein Weg des Umgangs mit dem intergenerationell traditierten Schuldtrauma sowohl auf Opfer- als auch auf Täterseite ist das Erzählen: Seit den 1990er Jahren formiert sich die sogenannte ,zweite Generation " nach der Shoah verstärkt auch als literarische Gruppierung (TEUFEL / SCHMITZ 2007:214) - in der österreichischen Literatur sind neben Robert Schindel, Doron Rabinovici noch Robert Menasse ${ }^{4}$ und Vladimir Vertlib ${ }^{5}$ als bekannte Vertreter dieser

4 Genauere Informationen zu den biographischen Kontexten von Robert Schindel, Doron Rabinovici und Robert Menasse bietet BeILEIN (2008:15-21). Auf die 
literarischen Formation zu nennen, obgleich die letzteren zwei, biographisch betrachtet, nicht unmittelbar als Nachgeborene von Shoah-Überlebenden bezeichnet werden können. Letztendlich aber scheint mir (neben der biographischen Komponente) die Thematik der Texte ausschlaggebend für die Zurechnung zu dieser literarischen Formation zu sein: Die Texte dieser Autoren setzen sich mit der Shoah, mit Erfahrungen ihrer Eltern bzw. ihrer Elterngeneration und mit deren und ihren eigenen Schuldtraumata auseinander dadurch suchen sie narrativ nach ihren eigenen (jüdischen) Identitäten durch Erinnerung. Es sind nicht die eigenen Erfahrungen, an die sie sich erinnern, daher ist ihre Literatur keine Zeugnisliteratur im Sinne der Überlebendenliteratur, sie bezeugt aber das Trauma einer Generation von Nachgeborenen (TEUFEL / SCHMITZ 2007: 215), die viel mit sich herumtragen und sich oftmals als lebende Museen empfinden, in welchen „die Eltern und Großeltern ihre Erinnerungen, Erfahrungen, Alpträume eingeschlossen haben. So wie sie sterben wir tausend Tode [...].“ (Janine Chasseguet-Smirgel, zit. nach: Teufel / SchMitz 2007: 213). Wenn im Folgenden der Text von Martin Pollack, dem Sohn eines NS-Verbrechers, neben den Texten von zwei ,Opferkindern" besprochen wird, so soll nicht der Unterschied ${ }^{6}$ zwischen den Schuldtraumata der Opfer und deren Nachkommen auf der einen Seite und der Täter und deren Nachkommen auf der anderen Seite verwischt werden und eine gemeinsame ,zweite Opfer-Täter-Generation' konstruiert werden: Es soll vielmehr aufgezeigt werden, dass, wie es Vertlib formuliert: „Verdrängung und Sprachlosigkeit“" etwas ist, ,was Täter, Opfer und deren Kinder oft gemeinsam haben." (Vladimir Vertlib, zit. nach TEUfEL / ScHMITZ 2007:215). Pollack hat mit seinem Buch Der Tote im Bunker gegen ein Klima

Frage, ob er sich als Jude fühle, antwortete Menasse: Nach den Nürnberger Rassegesetzen ja, nach seiner eigenen Einschätzung nicht. Menasse setzt sich allerdings als Literat sowohl in seiner Romanprosa als auch in seiner Essayistik mit der Shoah auseinander (STEINECKE 2006:247-251).

$5 \quad$ So setzt sich Vladimir Vertlibs Roman Letzter Wunsch (2003) direkt mit der Problematik der ,zweiten Generation' auseinander. Die Auseinandersetzung mit jüdischer Identität nach der Shoah, mit dem Zionismus, das Finden und Durchsetzen eigener Identität in Konfrontation mit den Identitätsentwürfen der Eltern sind Themen, die sich auch durch andere Werke Vertlibs ziehen.

6 Auf diesen Unterschied pocht etwa RabinOvici (2001:137), wenn er in Abhebung vom Umgang jener Österreicher mit der NS-Vergangenheit, die sich dieser stellen wollen, schreibt: „Die Empfindungen, die Schuldgefühle und Traumata, die in meiner Familie gegenüber dem Verbrechen vorherrschen, sind von anderem Charakter." 
der ,Verdrängung und Sprachlosigkeit', das in seiner Familie und darüber hinaus in vielen österreichischen Familien herrscht(e), angeschrieben. Er stellt sich seinem Schuldtrauma, wenn dieses auch anderer Art ist als das von Rabinovici und Schindel. Erzählen ist für alle drei im Folgenden zur Debatte stehenden Autoren der ,zweiten Generation“ auch ein Akt der Katharsis, der Psychohygiene, des Loswerdens von Belastungen durch Schuldtraumata - in diesem Sinn sind die Texte als psychonarrativ zu charakterisieren: Sie sind therapeutisch, indem sie dem (Ver-)Schweigen das Erzählen, dem NichtErinnern das Erinnern, dem Nicht-Kommunizieren das Kommunizieren entgegenstellen.

\section{Schuldverstrickte Identitäten zwischen Mullbinden: Doron Rabinovicis Suche nach M.}

Rabinovicis 1997 im Suhrkamp-Verlag erschienener Roman Suche nach M. bringt Schuldverstricktheit und Identitätssuche nach der Shoah schon durch seine bloße Komposition zum Ausdruck. Diese ist ebenso verwickelt wie die Identitäten seiner Figuren. Rabinovici, so schreibt WendELIN SCHMIDTDENGLER (1997:V) in seiner Rezension, verbindet zwölf Episoden mit unterschiedlichen Personen zu einem kohärenten Roman, ,[...] zaubert durch die jeweils neue Anordnung der Motive und Figuren kaleidoskopartig überraschende Konstellationen herbei und vermag so den Leser nicht nur mit einem dünnen Erzählfaden, sondern geradezu mit einer Packung von Mullbinden einzuwickeln.“ Es ist nicht notwendig, der Verwicklung der zwölf Handlungsstränge und der sich in sie verwickelnden Figuren des Romans im Detail nachzugehen - die oft überraschenden Auflösungen dieser Verwicklungen verleihen dem Roman ein detektivisches Moment, teilweise trägt der Text Züge eines spannenden Kriminalromans, wobei die ständige Präsenz des ,Unheimlichen', das nach Sigmund FREUd (1989:227-268) durch das Auftauchen von Verdrängtem generiert wird, dem Leser eine behagliche Unterhaltung verbietet. Das Verdrängte, das da auftaucht, ist die Verstrickung eines ganzen Landes, nämlich Österreichs, in eine unbewältigte, unaufgedeckte Schuld. Die zentrale Gestalt des Romans ist Mullemann, eine mit Mullbinden umwickelte Gestalt. Mullemann bekennt sich zu Verbrechen, die er selbst nicht begangen hat, mit dem Satz: „Ja, ich war's. Ich bin's gewesen. Ich bin schuld“ (RABINOVICI 1997:179) und weiß darüber hinaus auch noch über die genauen Umstände der von ihm eingestandenen Verbrechen Bescheid: Er wird ein „Schuldphantom“ genannt, „,on dem das ganze Land 
fiebert“, ein „Mummenschanz“, eine „Mumie“, ein „Dibbuk“ (RABINOVICI 1997:173, 178, 183 etc.) Wenn Mullemann zwar verschiedenste Verbrechen aufdeckt und sogar zur Überführung eines Frauenmörders beiträgt, geht es ihm doch nicht um den „Filz der Politik, um die Verstrickungen der Finanz, die Seilschaften des Parlaments, die das Volk seit Jahrzehnten lenkten und gängelten“ (RABINOVICI 1997:182), wie die österreichischen Väter meinen. Die österreichischen Großväter erraten schon eher, auf welche Schuldverstrickung Mullemann in erster Linie verweist:

Wer, so fragten einzelne Großväter mit zitternden Zeigefingern, wäre in der Stadt denn frei von Schuld? Wie viele Delikte, Streitigkeiten und Feindschaften seien doch bloß mit Mühe unter den Teppich gekehrt, wie viele Leichen unter der Erde verscharrt worden? Sollten all die Anstrengungen ihrer Generation vergeblich gewesen sein? Hatten sie nicht alle Spuren im Wiederaufbau verwischt, alle Erinnerungen mit verbissenem Schweigen ausgelöscht? (RABINOVICI 1997:182)

Mullemann ist nicht ein Verbrechensaufdecker mit detektivischem Impetus oder ein eifriger Korruptionsbekämpfer: Er handelt aus eigener Verstrickung in unbewältigte Schuld heraus, zu der er unschuldig gekommen ist. Er trägt ein Schuldtrauma in sich, das sich physisch in einem Hautausschlag niederschlägt, deshalb auch die Mullbinden. Hinter Mullemann verbirgt sich Dani Morgenthau, der Sohn von Mosche und Gitta Morgenthau, Überlebenden der Shoah in Polen.

Durch seine bloße Existenz steht Dani in der Schuld - dies wird besonders deutlich in einer Passage, in der Dani über die menschliche Fortpflanzung reflektiert, die für ihn ebenso ein Geheimnis bleibt wie die Erlebnisse der Eltern in der Zeit der nationalsozialistischen Verbrechensherrschaft: ,[...] daß er einer solchen Verquickung seiner Eltern entstammte [...]“ (RABINOVICI 1997:32), kann er sich ebenso wenig vorstellen, wie dass seine Eltern beim Verbrechen der Massenvernichtung dabei gewesen sind. Die Geographie des weiblichen Körpers bleibt für Dani ein Tabu ebenso wie die Anatomie des Leidensweges seiner Mutter, die Namen der Konzentrationslager, in denen seine Mutter und Großmutter gewesen sind, ebenso wie die der Geschlechtsorgane. Durch die geschlechtliche Vereinigung seiner Eltern (durch deren ,Verquickung', die onomatopoetisch auf ,Verstrickung ' verweist), aus der Dani hervorgeht, ist er in einen intergenerationellen Schuldzusammenhang verstrickt, den er schon als Kleinkind von sich zu weisen trachtet, indem er alles, was ihm die Mutter zwischen die Zähne zwängen will, ausspeit. Danis Vater, selbst voller Unsicherheit und belastet von seinen Erinnerungen an sein Überleben in einem Versteck am Stadtrand von Warschau, imaginiert 
seinem Sohn ein Leben, das unbelastet vom Grauen der Vergangenheit sein sollte: „Es war einmal ein kleiner Junge, und der hieß Dani.“ Dani aber protestiert: „Ich will eine richtige Geschichte.“ (RABINOVICI 1997:27) Die richtige Geschichte aber gibt es nicht, ,,[...] denn Schweigen war Teil der mündlichen Überlieferung seiner Familie“ (RABINOVICI 1997:35). Kommunikations- und Erzählverweigerung, das Schweigen über die Shoah in Danis Familie führen zu seiner Erkrankung, dazu, dass er zu einem Dibbuk, einem wandelnden Schuldtrauma wird, das durch seine bloße Existenz an die nicht aufgearbeitete Schuld der NS-Verbrechen in Österreich erinnert.

Mullemanns Umgang mit der Schuld ist, so sehr ihn die in Verbrechen Verwickelten als Aufdecker fürchten, der eines passiven Erleidens. Dies wird deutlich, wenn man Mullemann mit seinem ,Gegenstück', seinem Gegenüber, dem israelischen Geheimdienstagenten Arthur Bein konfrontiert: Dieser begegnet Mullemann zufällig in einem Wiener Krankenhaus, wo er eine Infektion, die er sich bei einem Ägyptenaufenthalt geholt hat, auskuriert. Mullemann morst dem im Nebenzimmer liegenden Arthur Bein, was dessen Verdacht erweckt, da er in Mullemanns Morsezeichen auch einige seiner eigenen ,Untaten“ erkennt. In einem Moment scheinen Arthur Beins und Mullemanns Identitäten fast zu einer Art Symbiose zu verschmelzen:

Vielleicht bin ich, ist Mullemann, kein Mensch aus Fleisch und Blut, vielleicht bin ich bloß von Verbänden und Kompressen durchdrungen, bin ich gänzlich in mich verschlungen. Zuweilen erscheint es mir, als wäre Mullemann ein Schmerzpaket zahlloser Tode; nichts als ein Erinnerungsbündel aus verschiedenen, zufällig verwobenen Mullrollen. In manchen Momenten denkt Mullemann: Vielleicht gibt es mich nicht. (RABINOVICI 1997:114)

Mullemann ist ein Verweis nicht nur auf eine Mumie, auf einen Dibbuk, sondern auf einen ,Muselmann“ - eine Bezeichnung, mit der sich KZ-Häftlinge auf ,die schwachen, untauglichen und selektionsreifen Häftlinge“ (LEVI 2009:105) bezogen. Einen Muselmann beschreibt der Shoah-Überlebende PRIMO LEVI (2009:108) als einen ,verhärmte(n) Mann mit gebeugter Stirn und gekrümmten Schultern, von dessen Gesicht und Augen man nicht die Spur eines Gedankens zu lesen vermag." Arthur Bein, ursprünglich Arieh Fandler, vermag Mullemanns Identität zu erkennen, weil auch seine Kindheit und Jugend im Schatten der Shoah und des Schweigens über sie steht: Auch Ariehs Existenz ist von Geburt an schuldbelastet: In ihm „saß eine geheimnisvolle Schuld, von der er nichts ahnte, die aber seinem bloßen Dasein, der Gegenwart schlechthin anhaftete" (RABINOVICI 1997:49). Sein Vater, Jakob Fandler, ein jüdischer Wiener Geschäftsmann, erzählt seinem Sohn Arieh nichts über sich und seine Vergangenheit. „Wer ich war, wird auf meinem 
Grabstein stehen. Ein wenig Geduld, mein Sohn.“ (RABINOVICI 1997:49) Schließlich erfährt Arieh allerdings doch, dass sein Vater in Wirklichkeit Jakov Scheinowiz geheißen habe und ein doppelter Doktor und Lektor der Germanistik in Krakau gewesen sei, dass er eine Frau und eine Tochter gehabt habe, die beide umgekommen seien, vom Krakauer Ghetto, von seiner Flucht unter dem Namen Fandler, davon, dass er im Nachkriegs-Wien einmal mit einem Buchdrucker Adam Kruzki, der im Ghetto ermordet worden sei, verwechselt worden sei. Dabei verschweigt er seinem Sohn jedoch, dass er mit diesem Buchdrucker bereits früher von einem SS-Mann im Ghetto verwechselt worden ist und nur deshalb überlebt hat. Er kommt im Gespräch mit seinem Freund Leon Fischer vor seinem Tod zu dem Schluss: „, [...] Mein ganzes Überleben war eine Verwechslung.““ (RABINOVICI 1997:101) Der Name Scheinowiz steht für Mimikry, Schein-Identität und im Letzten für ein als Schuld empfundenes Überleben, aus dem Arieh selbst entspringt.

Allerdings ist Ariehs Umgang mit dieser Schuldverstrickung dem von Mullemann konträr entgegengesetzt. Während Mullemann seine eigene Schuldverstrickung und die anderer erleidet und als Leidensgestalt zum Aufdecker wird, ist Ariehs Verhältnis zur Schuld von Aktivität und Offensivität geprägt - ein Kontrast, der bereits in der Erziehung der beiden grundgelegt ist: Während Danis Vater voller Unsicherheit jede Aussage in eine Frage umwandelt, also von vornherein in die Defensive geht, lässt Ariehs Vater seinen Sohn bereits als Kind die Kampfsportart Taekwando lernen: „,Dich soll keiner ungestraft schlagen, Arieh. Hörst du? Dich nicht ' [...].“ (RABINOVICI 1997:49) Man denkt an Amérys eindrucksvolle und erschütternde Aussage in seinem Aufsatz über die Tortur, nach der die Grenzen meines Körpers die Grenzen meines Ichs seien. Die Hautoberfläche schließe mich ab gegen die fremde Welt: auf ihr dürfe ich, wenn ich Vertrauen haben solle, nur zu spüren bekommen, was ich spüren wolle. Mit dem ersten Schlag aber breche dieses Weltvertrauen zusammen. Der andere zwinge mir mit dem Schlag seine Körperlichkeit auf (AMÉRY 1988:44f.). Der Schlag, der Ariehs Leben in andere Bahnen lenken sollte, gilt gar nicht ihm, sondern einem Afrikaner, der von einer Gruppe Rechtsradikaler in einem Wiener Vorstadtlokal angegriffen wird. Dem Studenten Arieh wird die Suche nach dem Schuldigen zur Sucht „,in einem Land, das allgemeine Unbeflecktheit beanspruchte“ (RABINOVICI 1997:47) - eine Anspielung auf den österreichischen Opfermythos, der besonders nach der Waldheim-Affäre im Jahre 1986 ins Wanken geriet und in Josef Haslingers Essayband Politik der Gefühle (1987), in Thomas Bernhards Heldenplatz (1988), in Doron Rabinovicis, Robert Schindels, Robert Menas- 
ses u.a. journalistischen, essayistischen und literarischen Texten ${ }^{7}$ einer fundamentalen Dekonstruktion unterzogen wurde.

Arieh gelingt es bald, den Hauptverantwortlichen für die Attacke gegen den Afrikaner ausfindig zu machen. Ohne es zu wollen, tötet er den rechtsradikalen jungen Mann. Er flieht nach Israel, nimmt den Namen Arthur Bein an und wird $\mathrm{zu}$ einem Agenten des israelischen Geheimdienstes. Im Dienste der zionistischen Utopie, die im Staat Israel Wirklichkeit geworden ist, sucht er „,nach den Todfeinden ihrer Existenz“ (RABINOVICI 1997:142). Im Essay Im Widerschein Israels. Oder Der jüdische Staat in der Diaspora schreibt RABINOVICI (2001:56):

Allein der Mythos von einem schlagkräftigen Geheimdienst, das Wissen um eine Armee, in der die Kohns und Levis Generäle werden, änderten das Selbstwertgefühl aller Juden. Nachdem Adolf Eichmann aus Argentinien entführt und in Jerusaelm abgeurteilt worden war, bangten die Mörder in allen Ländern um ihr Leben. Nun mußten sie erkennen, daß Juden nicht mehr Freiwild und ewige Opfer waren. Der Fluch der Verfolgung war gebrochen.

Arieh ist im Unterschied zu Mullemann keine duldende Märtyrergestalt, sondern Teil einer schlag-kräftigen Organisation. Doch gerade in seiner Funktion als Geheimdienstagent setzt er die Mimikry seines Vaters, das Spiel mit Schein-Identitäten fort. Er wechselt die Namen sogar öfter, als es sein Vater je getan hat. Ebenso wie sein Vater macht auch er sich vor seiner Tochter zum Geheimnis.

Mullemann und Arieh sind zwei verschiedene Figurationen jener, zweiten Generation ', der Nachgeborenen von Shoah-Opfern. Sie sind beide zu dem geworden, was sie sind, durch eine aus einem Schuldtrauma entsprungene Haltung der Erzähl- und Kommunikationsverweigerung. Ob Arieh aus seinen Schein-Identitäten zu seiner Identität und Mullemann, dem Sophokles'schen Ödipus ähnlich, zur a-letheia (,Wahrheit'), zu einem Zustand des Entborgenseins, des aus der Verhüllung Heraustretens finden kann, muss dahingestellt bleiben. Mullemann kann aber als „Wahrheitsdetektor“ (SCHMIDT-DENGLER 1997:V) dazu beitragen, dass eine Wahrheit zumindest ans Tageslicht kommt: „Was ein Jude ist und was ein Österreicher, das ist seit 1945 neu definiert." (RABINOVICI 2001:137) Der Anspruch einer ,allgemeinen österreichischen Unbeflecktheit' ist im Angesicht von Mullemann jedenfalls nicht mehr möglich.

7 Dazu detailliert für Schindel und Rabinovici BEILEIN (2008:23-129). 
Alexander Höllwerth

\section{Zwischen Radetzkymarsch und Todesfuge: Robert Schindels Gebürtig}

„Die Gerechtigkeit, das ist ja ein groteskes Ideal.“(SCHINDEL 1992:145), sagt der in New York lebende Erfolgsautor Hermann Gebirtig, eine Figur aus Schindels 1992 im Suhrkamp-Verlag erschienenen Roman Gebürtig. Er soll nach Österreich, in dieses „Naziland“ fahren - „,was Juden heute in Österreich zu suchen haben“ (SCHINDEL 1992:121), versteht er nicht -, um als ehemaliger Häftling des KZ Ebensee den sadistischen KZ-Aufseher Anton Egger, den sogenannten ,Schädelknacker', der als Albert Eigler ein unbehelligtes Leben als Pensionist seinen Lebensabend genießt, zu identifizieren. Erkannt wurde der ,Schädelknacker" vom Vater der Journalistin Susanne Ressel, der als kommunistischer Spanienkämpfer, als sogenannter ,Spaniak‘, ebenfalls im KZ Ebensee inhaftiert war. Susanne gelingt es schließlich, mit Hilfe von David Lebensart, in dem nicht unschwer der als Nazijäger apostrophierte Simon Wiesenthal zu erkennen ist, Hermann Gebirtig gegen dessen innere Widerstände davon zu überzeugen, dass es ,seine verdammte Schuldigkeit“" sei, ,dafür zu sorgen, daß jeder, der Blut an seinen Händen hat, verurteilt wird“ (SCHINDEL 1992:171). Dass nun Magister Wendelin Katzenbeißer, Sekretär des Wiener Kulturstadtrates Eugen Trnka, eine Ehrung des Wiener Emigranten Gebirtig durch die Stadt Wien - „eine Versöhnung zwischen Wien und Gebirtig“ (SCHINDEL 1992:207) - zustandebringen möchte, obwohl sich Gebirtig extra auserbeten hat, dass er nur im Prozess auszusagen und dann wieder nach New York zurückzufliegen gedenke und keinerlei Aufmerksamkeit vonseiten der Öffentlichkeit wünsche, verleiht dem Aufenthalt Gebirtigs in Wien einen komödiantisch-farcehaften Charakter. Eine Farce, so fürchtet Susanne Ressel, könnte auch der Prozess gegen Egger werden, da die Verteidiger versuchen würden, die höchst traumatisierten Belastungszeugen durch Fangfragen durcheinanderzubringen - ein ,geordneter Diskurs von Anklage, Verteidigung und Urteilsspruch“im Sinne von Arendt ist damit von vornherein ausgeschlossen. Angesichts des Freispruchs des Angeklagten durch ein Geschworenen-Gericht findet sowohl Gebirtigs Gerechtigkeitspessimismus als auch Susanne Ressels Befürchtung eine Bestätigung. Die Folge des Freispruchs ist der sofortige Rückflug Gebirtigs, dem Österreich und Wien gerade wieder zu gefallen angefangen haben, nach New York. 
Dies ist freilich nur ein Handlungsstrang der viele Erzählebenen ineinander verschachtelnden Schindelschen Romankomposition, die in den Rezensionen teilweise gelobt, teilweise getadelt wird ${ }^{8}$ - er stammt aus einem Manuskript des Wiener Autors Emmanuel Katz, das der Lektor Danny Demant zur Begutachtung bekommt, dieser wiederum hat einen Zwillingsbruder Alexander, der die Geschichte Dannys erzählt. Hauptschauplätze dieser Erzählebene sind die Wiener Beisel und Kaffeehäuser, in denen sich Danny Demant in den 1980er Jahren mit seinen jüdischen und nicht-jüdischen Freunden trifft. Ebenso wie in Ingeborg Bachmanns Erzählung Unter Mördern und Irren (1961) kann auch hier eine richtige Kaffeehaus-Gemütlichkeit nicht aufkommen, aber aus anderen Gründen. Während sich in Bachmanns Erzählung die männlichen Teilnehmer an NS-Verbrechen treffen, geraten in Schindels Kaffeehäusern die Kinder der Opfer der NS-Verbrechen (Demant, Singer, Katz) mit ihren österreichischen Freunden wegen unterschiedlicher Zugänge zur Vergangenheit in Streit: Ein Anlass eines solchen Streites ergibt sich etwa, als der oberösterreichische blonde Freund der jüdisch-wienerischen Soziologin Mascha Singer meint, er habe als Kind immer auf dem Gelände des Konzentrationslagers Mauthausen gespielt. „Ein Superspielplatz.“ (SCHINDEL 1992:10) Ebenso wenig wie Kaffeehaus-Gemütlichkeit lässt Schindels Roman einen nostalgischen Bezug auf Österreichs Habsburg-Vergangenheit unter Ausblendung der NS-Vergangenheit zu. Durch den Namen Demant spielt Schindel auf den jüdischen Regimentsarzt einerseits auf Dr. Demant aus Joseph Roths Roman Radetzkymarsch (1932) an, andererseits lässt sich auch ein Bezug zu Dora Diamant, der letzten Frau in Kafkas Leben, herstellen: Dora Diamant, eine polnische Jüdin, floh vor den Nazis in die Sowjetunion, während Milena Jesenká, Greta Bloch ebenso wie Kafkas drei Schwestern dem Naziterror zum Opfer fielen (GLATZER 1987). Zwischen Roths Radetzkymarsch und Schindels Gebürtig liegt nicht nur der endgültige Zusammenbruch des Habsburgischen Vielvölkerreiches, sondern auch die Vernichtung der Juden - eine Tatsache, die schon im Prolog des Romans, der den Titel ,Doppellamm“ trägt, deutlich wird. ANDRZEJ KOPACKI (2009:48) sieht im ,Doppellamm` zu Recht eine Metapher für Juden in Österreich,

8 Um nur zwei Beispiele aus den zahlreichen Rezensionen herauszugreifen: WALTER VOGEL (1992:VII) spricht in seiner Rezension für die Presse von einem „,in seiner Vertracktheit schließlich extrem klarem, in seiner Kompliziertheit so einfachem Buch“, WOLFGANG HöBEL (1992:II) kritisiert in der Süddeutschen Zeitung die Romankonstruktion trotz einer insgesamt positiven Rezension als „mitunter ärgerlich überladen“. 
Doppellamm verweist auf Doppeladler ${ }^{9}$, das Wappentier des Habsburger Vielvölkerreiches: „Die Kinder des Doppeladlers waren in ihrer Mehrheit Schafe, die ihr Leben damit verbrachten, sich in Scheiße und Blut zu wälzen, so daß ihre letztendliche Schlachtung keinen sättigte, außer Gott. Deren Kinder sind wir, sagte der Lektor Demant zu seinem Herzen [...]." (SCHINDEL 1992:207) Diese Stelle lässt sich mit dem ,Holocaust" (üblicherweise als ,Brandopfer' übersetzt) assoziieren: Der Begriff stammt vom lateinischen Substantiv ,holocaustum', das eine Übersetzung des griechischen Adjektivs ,holokaustos“ ist; ursprünglich bezog sich dieses Wort auf die Opferung von Tieren an Gott. Dessen Übertragung auf die Vernichtung der Juden ist nicht unproblematisch, da die Bedeutungsgeschichte des Begriffs ,Holocaust ' im Wesentlichen eine christliche ist, und der Begriff wurde häufig auch in einem antisemitischen Kontext verwendet. AGAMBEN (2003:25-28) kritisiert an dem Begriff, dass er suggeriere, die Vernichtung der Juden als Opfer an Gott habe doch einen Sinn gehabt - Demants Gedanke, dass die Schlachtung der ,Schafe “ keinen außer Gott gesättigt habe, spielt auf die letztendliche Sinnlosigkeit der Vernichtung der Juden an. Der Prolog schließt mit einem Gedicht (SCHINDEL 1992:16), das einen intertextuellen Bezug auf Paul Celans Todesfuge (1952) aufweist:

Weit im Himmel das Luftgrab

Der sechs Millionen Lämmer

Das Doppellamm fragt jetzt um Rat

Das Land der Söhne, Land der Hämmer.

Die Zeile aus Celans Todesfuge ,wir schaufeln ein Grab in den Lüften da liegt man nicht eng" (CELAN 1970:18) wird zur österreichischen Bundeshymne ,[...] Land der Hämmer, zukunftsreich! Heimat bist du großer Söhne [...]“ in Bezug gesetzt. Die Hämmer, die für Fleiß und Arbeit stehen, können, indem sie sich auf die sechs Millionen Lämmer (die Opfer der Shoah) reimen, als eine Metapher für ,Mordwerkzeuge' verstanden werden. Damit stellt Schindel von vornherein klar, dass jene historische Rekonstruktion und Idealisierung einer österreichischen Vergangenheit, wie sie der ,Epiker und enttäuschte Nostalgiker' Joseph Roth nach dem Zusammenbruch der Habsburgermonarchie im Radetzkymarsch versuchte (KASZYŃSKI 2006:93-107), nach dem ,Zivilisationsbruch Auschwitz' nicht mehr möglich ist. Dies wird deutlich in einem Gespräch zwischen der Ärztin Christiane Kalteisen, der

9 Peter Diem (1995:109f.) weist darauf hin, dass das zum ,Archetyp“ gewordene altösterreichische Staatssymbol des Doppeladlers oft unter dem Vorzeichen einer Habsburg-Nostalgie revitalisiert wird. 
scheinbar von der Vergangenheit der Judenvernichtung unberührten österreichischen Freundin Danny Demants, und dem traumatisierten Emmanuel Katz, dessen Mutter drei Jahre in Birkenau gewesen ist:

„Frau Doktor“, sagte Katz, „,von Auschwitz verstehen Sie nichts.“

„Es lag in Galizien.“

„Es liegt immer noch dort.“ (SCHINDEL 1992:196)

Und dennoch gibt es kein Zurück nach Galizien, in den von Joseph Roth mythisierten ,Osten ' des Habsburgischen Vielvölkerreiches. Dies wissen jene Protagonisten von Schindels Roman, deren eigene Identitätssicherheit von der Vergangenheit massiv belastet ist: Dies sind etwa Emmanuel Katz, der den Großteil seines Lebens nichts anderes getan hat, ,als seiner todwunden Familie umfassendes Auffangbecken für deren Melancholie zu sein" (SCHINDEL 1992:2), und der Kulturjournalist Konrad Sachs, der von einer dibbukhaften Gestalt, dem ,Prinzen von Polen', besessen ist. Schindels Romanfigur Konrad Sachs ist eine Hommage an den Stern-Reporter Niklas Frank (geb. 1939), den Sohn des Genaralgouverneurs des von Hitlers Wehrmacht überfallenen Polen, Hans Frank, des ,Königs von Polen', der im Roman als Ernst Sachs figuriert. Es sind Erinnerungen an Leichenberge, die Konrad Sachs als Fünfjähriger an der Hand seines Vaters sah, und an den Leichengeruch, den er in der Nase hatte, die im ,Prinzen von Polen ' Gestalt annehmen und ein Eigenleben entfalten, die sein nach außen geordnetes Leben, seine Ehe mit Else immer mehr bedrohen. Konrad Sachs, der Angst davor hat, sich als Sohn eines im Nürnberger Prozess zum Tod verurteilten und hingerichteten Kriegsverbrechers zu ,outen', weiß nicht, wie er seine Last loswerden kann. Wenn Konrad Sachs beim Gedanken an die Hinrichtung seines Vaters onaniert (dabei folgt Schindel übrigens genau der literarischen Vorlage seiner Figur) ${ }^{10}$ und in Gegenwart einer Prostituierten, der er in einem Hotelzimmer seine Herkunft und Identität beichtet (SCHINDEL 1992: 188, 233), auf der Toilette seine Gedärme entleert, so werden Ejakulation und Exkrementation zu Metaphern für den Drang, sich von einer verhassten Vaterfigur und von belastenden Erinnerungen zu befreien. Wirkliche Befreiung von seinem Dibbuk, der die ganze Zeit „Uwaga“ (,Achtung') schreit, erhofft sich Konrad Sachs von

10 In seinem Buch Der Vater. Eine Abrechnung schreibt NIKLAS FRANK (1987:8): „Ich mochte Dein Sterben. Ich legte mich nackt hin, die Beine gespreizt, die Linke am schlaffen Glied, und mit einer leichten Rubbelbewegung fing ich an Dich zu sehen, wie Du auf und ab gehst in Deiner Zelle [...]." Vgl. dazu Schindels Darstellung: „Hernach nahm er sein Geschlecht in die Hand. Sein Vater wurde zur Hinrichtung geführt.“ (SCHINDEL 1992:188) 
Alexander Höllwerth

Emmanuel Katz, dem er auf der Nordseeinsel Borkum zufällig begegnet und den er brieflich um eine Aussprache bietet. Dieser schickt Sachs seinen Freund Danny Demant, der ihm empfiehlt, seinen ,Prinzen von Polen' hinauszuwerfen und an die Öffentlichkeit zu zerren. Diesen Rat befolgt Sachs entgegen anfänglichem Widerstreben und veröffentlicht ein Buch mit dem Titel „Uwaga, der Prinz von Polen“ - darüber hinaus möchte er noch mit Hilfe einer Psychoanalyse bei einem jüdischen Analytiker eine Reise in seine Kindheit unternehmen. Schreiben und Publizieren als kathartische, exorzistische Akte stellen sich bei Robert Schindel gegen Verdrängung und Verschweigen und gehen Hand in Hand mit einer Psychoanalyse.

\section{Zwischen Orientalisierung und Orientierung: Martin Pollacks Der Tote im Bunker}

Mit seinem Buch Nach Galizien. Von Chassiden, Huzulen, Polen und Ruthenen (1994) verführt der Slawist und Historiker Martin Pollack seine Leser zu einer imaginären Reise durch die verschwundene Welt Ostgaliziens und der Bukowina - er dokumentiert seine Reise kenntnisreich mit Materialien, Fotos, Landkarten. Auch Pollacks Buch atmet jene Nostalgie oder Melancholie, die aus Joseph Roths literarischen Darstellungen bekannt ist, wenn es auch ganz anders als diese geschrieben ist. Die Routen und Wege, auf denen dieses Buch führen soll, sind fiktiv - müssen es sein, weil es die ethnische und kulturelle Vielfalt, die jene Welt ausmachte, nicht mehr gibt (POLLACK 1994:7). In einem in der Gazeta Wyborcza abgedruckten Interview gibt POLLACK (2005) zu, dass auch er einen Beitrag zum Mythos der schönen galizischen Provinz geleistet habe, dass er aber schließlich die andere, dunkle Seite Mitteleuropas zeigen musste. Das provinzielle Grenzland habe nicht nur Kafka, Schulze, Freud und die Maler der Sezession hervorgebracht, sondern auch jene, die diese Welt zerstören wollten. Dort eben sei die nationalsozialistische Ideologie entstanden. Pollack spricht dann von der österreichischen Provinz, vom multiethnischen Wien, in dem Hitler seinen Antisemitismus erworben habe, von Ernst Kaltenbrunner, der aus Linz stammt, von Alois Brunner und Adolf Eichmann, ranghohen Funktionären der Vernichtungsmaschinerie des NSRegimes, die allesamt der österreichischen Provinz entsprangen (POLLACK 2005) - einer dieser Funktionäre war der 1911 geborene ehemalige SS-Sturmbannführer Dr. Gerhard Bast, im März 1947 am Brenner ermordet, an der österreichisch-italienischen Grenze, auf dem Weg nach Innsbruck, von wo aus er sich vermutlich mit Frau und Sohn nach Übersee absetzen wollte. 
Pollacks 2004 im Wiener Zsolnay-Verlag erschienenes Buch Der Tote im Bunker ist dem Leben dieses Mannes gewidmet - der Untertitel des Buches lautet Bericht über meinen Vater. „Und wieder einmal möchte ein Sohn verstehen, was war und wie es dazu kommen konnte“, schreibt ERNA LACKNER (2004:L16) in der FAZ. Pollacks Vater wurde von einem ,normalen Kriminellen ermordet, bevor die Justiz seiner habhaft werden konnte. Er musste nicht im Sinne von Hannah Arendt in den ,geordneten Diskurs von Anklage, Verteidigung und Urteilsspruch' treten, wie dies Niklas Franks Vater Hans Frank, der Generalgouverneur von Polen, tun musste und sich vor Gericht der Frage stellen, warum gerade er ,ein Funktionär in diesem System geworden sei ‘. Pollack kann nicht wie Niklas Frank in Gerichtsakten nachlesen, wie sein Vater selbst seine Taten im gerichtlichen Diskurs erklärt bzw. rechtfertigt. Er kann nicht wie Niklas Frank in Der Vater. Eine Abrechnung (1987) auf umfangreiche Tagebuchaufzeichnungen eines sich als humanistisch-gebildet gerierenden Vaters zurückgreifen, mit dem er in einen emotionalen und anklagenden Dialog treten und in Du-Form abrechnen kann.

Pollack dagegen ist mit einem sprachlosen ,Toten im Bunker' konfrontiert, mit dem er nicht abrechnen kann, sondern den er sich erst durch harte Recherchearbeit in Archiven erarbeiten muss, zu dem er nicht ,Du' sagen kann, weil er ihn nicht kennt. Er anerkennt ihn aber als ,mein Vater', was die Distanz zum Vater nicht aufhebt, weil er für ihn ein Fremder, ein Verborgener, ein Pater absconditus, bleibt: „Über das private Leben meines Vaters, über das, wie er war, wie er dachte, weiß ich wenig, eigentlich nichts. Er ist für mich bis heute im dunkeln geblieben, eine Figur, von der ich nur undeutliche Konturen erkenne." (POLLACK 2004:121) Aus dieser Fremdheit des Vaters folgt auch der distanziert-sachliche Ton, in dem Pollacks Text geschrieben ist. Hinter diesem Ton klingt jedoch manchmal ein anderer Ton hervor, der einem inneren Ringen mit dem verborgenen Vater zu entspringen scheint. Dies geschieht etwa, wenn POLLACK (2004:208f.) davon erzählt, wie ihm seine Mutter mit vierzehn einen Brief gegeben habe, vermutlich den einzigen, den sein Vater an ihn geschrieben habe: ,[...] eine kleine Bildgeschichte, niedergeschrieben und gemalt für den Sohn, der nicht lesen konnte, zur Erinnerung an den liebenden Vater. Irgendwann habe ich den Brief in einem Anfall von Verzweiflung, oder war es Wut?, verbrannt." Pollacks Bericht unterscheidet sich jedoch von einem amtlichen Bericht, dessen Aufgabe in der kommentarlosen behördlichen Dokumentation besteht: Die nüchtern-klare Sprache, in der dieser Bericht verfasst ist, ebenso wie seine Komposition sind als literarisch zu kennzeichnen: In Pollacks Text sind drei Erzählebenen ineinander verschachtelt. Auf der ersten Ebene, die den ande- 
Alexander Höllwerth

ren zwei Erzählebenen den Rahmen verleiht, erzählt der Ich-Erzähler, der mit dem Autor Pollack identisch ist, über die Recherchereisen, die er unternommen hat, um die Geschichte seines Vaters erzählen zu können, und seine Eindrücke während dieser Reisen. Auf einer zweiten Ebene wird die Geschichte des Vaters, das Resultat der Recherchearbeiten, erzählt, während die dritte Erzählebene die Familiengeschichte nach dem gewaltsamen Tod des Vaters, die Beziehungen Pollacks zu Mutter und Stiefvater in Linz einerseits und zu den Großeltern väterlicherseits in Amstetten andererseits thematisiert. Die drei Ebenen sind (dia-)logisch aufeinander bezogen. Der Text Pollacks stellt sich der tiefen Sprachlosigkeit entgegen, die der Autor selbst lange Zeit für Normalität gehalten habe (POLLACK 2004:185) - der Blockierung des ,kommunikativen Gedächtnisses` an den Vater durch Erzähl- und Kommunikationsverweigerung: Das Schweigen der Mutter ist u. a. durch die ,verwickelten familiären Verhältnisse“ (POLLACK 2004:123) und die Anwesenheit des Stiefvaters, von dem sie sich wegen des leiblichen Vaters Pollacks für kurze Zeit trennte, motiviert. POLLACK (2004:185) fragt: „Erfüllte sie mit ihrem Schweigen das Vermächtnis des toten Vaters?“ Auf der anderen Seite stehen die Großeltern aus Amstetten, vor allem die Großmutter, die die Anständigkeit und den Idealismus des Vaters betont: „Wir haben nie etwas getan, wofür wir uns schämen müßten, sagte sie noch [...].“ (POLLACK 2004:104) Pollacks Text kehrt immer wieder zur Person der Großmutter zurück, von der der Onkel sagt, sie sei ,gestorben wie eine deutsche Frau“ (POLLACK 2004:174). Die Großmutter reagiert mit Unverständnis auf Pollacks Entschluss, Slawistik und osteuropäische Geschichte zu studieren. Sie begreife nicht, woher die Liebe ihres Enkels zu den Slawen stamme. Die Vorstellung, ihr Sohn könne eine Polin oder gar eine Jüdin heiraten, ist für sie schlimm. Er müsse ihr beim Andenken seines Vaters versprechen, das nie zu tun. Wenn auch Pollacks Studienwahl ursprünglich von seiner Freundschaft mit den für ihn immer ,exotisch' und ,fremd' bleibenden Russen während der Besatzungszeit in Österreich motiviert ist, führt ihn seine „Neugierde für alles, was im Osten lag“ (POLLACK 2004:172) letztendlich in Regionen verdrängter Familiengeschichte.

In Schindels Gebürtig findet zwischen dem kleinen Konrad Sachs und Eggenberger, einem nationalsozialistischen Kameraden seines Vaters, bereits nach dem Krieg folgender Dialog statt:

„Jawoll, ich bin der Prinz von Polen. Mama, wo liegt Polen?“

„Im Osten“, antwortete der Eggenberger und gab ihm Schokolade.

„Und wo sind wir?“"

„Im Westen. Gott sei Dank, im Westen.“ (SCHINDEL 1992:53) 
Der Osten als Ort des Verbrechens wird im Sinne von Edward Saids Orientalismus (1978) ,orientalisiert‘, zum , wilden Osten` erklärt; das Verbrechen, der ,Zivilisationsbruch Auschwitz' und die eigene Schuld wird in den Osten ausgelagert, ,exterritorialisiert' und verdrängt: Während der , arme Christ" in Czesław Miłosz' Gedicht Biedny chrześcijanin patrzy na getto [Armer Christ schaut auf das Ghetto, 1945), auf das sich der Titel des Aufsatzes Biedni Polacy patrza na getto [Die armen Polen schauen auf das Ghetto, 1987) des polnischen Literaturwissenschaftlers Jan Błoński bezieht, es nicht so leicht hat, wegzuschauen von dem Verbrechen, das unter deutschem Besatzungsrecht in unmittelbarer Nachbarschaft passiert, transferiert (verdrängt) die Figur aus Schindels Roman das Verbrechen in den Osten und lokalisiert seinen eigenen Standpunkt im Westen: Durch die Orientalisierung des Verbrechens will Eggenberger eine Exkulpierung erreichen. Wenn SAID (2004) in seinem Vortrag Freud und das Nicht-Europäische den Versuch unternimmt, zwischen der psychoanalytischen Kategorie der Verdrängung und der kulturellen Ausgrenzung eine Verbindung herzustellen und Freuds Verdienst in einer Neukartographierung ,,angenommener oder überkommener Geographie und Genealogie" (SAID 2004:34) sieht, so charakterisiert er damit in gewisser Weise auch den Weg Pollacks - ich spreche bewusst von Weg, nicht von Methode, da Pollack nicht von Anfang an deutlich weiß, dass ihn seine „Neugierde für alles, was im Osten lag“, zur verdrängten Schuld seines Vaters führen würde, der ein Sonderkommando in Polen geleitet hat, unter dem es Geiselerschießungen gab:

Von dieser Geiselerschießung, ja vom Einsatz des von meinem Vater geleiteten Sonderkommandos in Polen erfuhr ich erst während der Arbeit an diesem Buch, nachdem ich mich vierzig Jahre lang mit Polen beschäftigt habe. Ich habe in Warschau studiert und übersetze polnische Literatur. Es sei ihr ein Rätsel, sagte meine Großmutter manchmal, weshalb ich mich ausgerechnet für Polen interessiere, für polnische Sprache und Literatur, was mich bloß darauf gebracht habe jetzt verstehe ich den ungläubigen Ausdruck, den sie dabei aufsetzte. (PollaCK 2004:204f.)

Bei seinen Recherchen über die Einsätze seines Vaters stößt Martin Pollack auf die Photographie eines Leichenbergs beim Berg Medzihrádky in der Slowakei, den er als bizarres Stillleben von zufällig oder mit Absicht sternförmig angeordneten Köpfen beschreibt: In dessen Zentrum ,[...] sieht man das Gesicht einer hübschen jungen Frau mit dunklen Haaren, von der man meinen könnte, sie schlafe friedlich, so gelöst scheint ihr Ausdruck, läge da nicht ein anderer Toter quer über ihrer Brust, den Mund weit aufgerissen in einem stummen Schrei.“ (POLLACK 2004:223) Zum ersten Mal, schreibt Pollack, bekommen die Opfer seines Vaters für ihn ein Gesicht. Die ,Neukarto- 
Alexander Höllwerth

graphierung angenommener oder überkommener familiärer Geographie und Genealogie" führt Pollack in die österreichische Provinz, in die Untersteiermark, eine Gegend, ,wo das Zusammenleben von Deutschen und Slowenen schon früh durch einen rabiaten Nationalismus vergiftet wurde" (POLLACK 2004:222). Sein Großvater stammt aus Tüffer, einem kleinen Marktflecken, der in jenem Teil des steirischen Kronlandes gelegen ist, der nach 1918 an Jugoslawien gefallen ist. Dorthin war sein Urgroßvater Paul Bast aus dem Rheinland gezogen. „Die Deutschen nannten sich Sprachgrenzdeutsche, das klang kämpferisch, wie Donnerhall.“ (POLLACK 2004:21) Auch wenn es in der Familie Bast Slowenen und Deutsche, sogar einen Halbjuden namens Guido gab, die sich bei Familienfeiern trafen, wird die Grenze zwischen Deutschen und Slawen auf der Ebene der Ideologie nie aufgehoben. Pollacks Text lässt keinen Zweifel daran, dass es die in der Untersteiermark herrschende ideologische Polarisierung war, die dazu führte, dass sich sein Vater für den Nationalsozialismus begeisterte. Er lässt aber ebenso keinen Zweifel daran, dass sein Vater, um es mit Bachmann zu formulieren, zu den „Mördern und Irren“ gehörte, die für die Ermordung von Millionen von Menschen verantwortlich sind. Damit bleibt freilich die Frage offen: Wie war der Vater menschlich dazu in der Lage, im Dienste einer irren Rassenideologie zu morden, wo er doch aus seiner Familie hätte wissen müssen, dass Slawen und Juden ,ganz normale Menschen" sind? Der Vater, der ihm von seinen Großeltern als lebensfroher, fescher Mann beschrieben wurde und auch auf Fotografien keinen unsympathischen Eindruck macht? Der Vater sei, so ein Mitglied seines Sonderkommandos Jahrzehnte später, stets human verfahren. „Human. Was bedeutete das in der Sprache der Täter?“ (POLLACK 2004:221) Eine Frage, die einem Sohn gestattet sein muss, ohne als Versuch einer Rechtfertigung oder gar Exkulpierung des Vaters ausgelegt zu werden, zumal in ihr schon die Skepsis mitklingt: Wenn es etwa heißt, die ,Zentralstelle" in Prag habe die Juden ,betreut", so bedeutet ,betreuen", in der Sprache der Täter“ in diesem Kontext „deportieren und töten“" (SCHMITZ-BERNING 2000:92f.).

Für Jakob Fandler aus Rabinovicis Suche nach $M$. ist Polen der Ort seiner Leidensgeschichte, aber auch der Ort, an dem sein Sohn Arieh eine Antwort auf die Frage hätte finden können, wer sein Vater und damit auch wer er selbst als Sohn eines Shoah-Überlebenden ist - diese Verquickung der Identität des Sohnes mit der des Vaters bringt Jakob Fandler mit einer Weisheit, die er im Cheder, der jüdischen Elementarschule, gelernt hat, zum Ausdruck: „Wenn du du bist, weil du du bist, und ich ich bin, weil ich ich bin, dann bist du du, und ich bin ich; wenn aber du du bist, weil ich ich bin, und ich ich bin, 
weil du du bist, dann bist du nicht du, und ich bin nicht ich." (RABINOVICI 1997:59) Als der todkranke Jakob Fandler, der in Polen Jakov Scheinowiz war, sein Versagen als Vater seinem Sohn Arieh gegenüber, der ohne ihn auf die Suche nach Scheinowiz gegangen sei, bedauert, empfiehlt ihm sein Freund Leon Fischer, er solle mit Arieh nach Krakau fahren und ihm die Lager zeigen (RABINOVICI 1997:97). Polen, der „Schauplatz“11 der Vernichtung, wird für die Opfer und deren Nachgeborenen zu einem Pol der Selbstverortung, der Identitätsfindung, der ,Orient-ierung ' (der Ausrichtung nach dem ,Osten '): ,Nach Krakau fahren' bedeutet, sich seiner Leidensgeschichte zu stellen, damit aufzuhören, sie zu verdrängen und dadurch sich selbst zu finden. Für den Journalisten und Osteuropaexperten ${ }^{12}$ Pollack bedeutet die Beschäftigung mit dem europäischen ,Osten " eine Orientierung in einem Raum, in dem Chassiden, Huzulen, Polen und Ruthenen zusammenlebten und gleichzeitig eine Distanzierung von den in seiner Familie vorherrschenden Vorurteilen gegen Juden und Slawen. Als Slawist tritt er in den polnischen „Klangraum“ (RABINOVICI 1997:15), in dem auch die Protagonisten von Rabinovicis Suche nach M. einst gelebt hatten. Als Übersetzer von polnischer Literatur ins Deutsche ${ }^{13}$ hilft er, Sprachgrenzen und ideologische Barrieren zu überwinden. Diese sprachliche und geographisch-kulturelle Orientierungsfähigkeit hilft Pollack dabei, in seinem Text Der Tote im Bunker den ,Tatorten' seines Vaters nachzugehen und damit seine eigene Familiengeschichte neu zu ,kartographieren' und sich gegenüber dieser Familiengeschichte zu positionieren und sich, indem er sich der Schuldverstrickung der Familie stellt, gegenüber dieser abzugrenzen.

11 BARBARA BREYSACH (2005:25) macht in ihrer breitangelegten komparatistischen Studie Schauplatz und Gedächtnisraum Polen. Die Vernichtung der Juden in der deutschen und polnischen Literatur darauf aufmerksam, dass sie sowohl Bezugspunkte jüdischer Leidensgeschichte als auch ,deutscher' Schuld sind.

12 Einige der Reportagen Pollacks, die sich direkt mit den Verbrechen des NSRegimes und speziell mit der Vernichtung der Juden befassen, sind in Buchform unter dem Titel Warum wurden die Stanistaws erschossen? 2008 im ZsolnayVerlag erschienen.

13 Martin Pollack übersetzte etwa Henryk Grynbergs Drohobycz, Drohobycz (poln. 1997, dt. 2000) oder auch Jarosław M. Rymkiewicz' Umschlagplatz (poln. 1988, dt. 1993), um nur zwei der bekanntesten Titel herauszugreifen, ins Deutsche. 
Alexander Höllwerth

\section{Fazit}

Alle drei besprochenen Romane setzen sich mit Schuldverstrickung und Identitätssuche angesichts der Shoah auseinander. Alle drei Romane wenden sich der bis 1986, dem Jahr der Waldheim-Affäre, ins Unter- und Unbewusste verdrängten, in den ,Osten ' abgedrängten österreichischen Schuld am ,Zivilisationsbruch Auschwitz‘ zu. In Rabinovicis Suche nach M. ist es Mullemann, in dem verdrängte Schuld eine dibbukhafte Verkörperung findet: Mullemann, der traumatisierte Nachgeborene von Shoah-Überlebenden, die Leidensgestalt. Sein Gegenbild, Arieh, ebenso traumatisiert, sucht einen anderen Umgang mit der Schuldverstrickung - aktives Zuschlagen und Zurückschlagen als Emanizipation aus der Rolle des Leidenden. Auf andere Weise als Mullemann und Arieh sind auch Emmanuel Katz und Konrad Sachs in Schindels Gebürtig aufeinander bezogen - Sachs als von seinem Vater, dem Generalgouverneur von Polen, Besessener erhofft sich von Katz, dem Auffangbecken der ,Überlebensschuld ' seiner Familie, eine Art ,Exorzismus', eine psychohygienische Katharsis. Pollack muss in seinem Buch Der Tote im Bunker seine Beziehung zum Vater, dem Leiter eines Sonderkommandos in Polen, erst bestimmen: Er geht einen Weg von der ,Orientalisierung ' der Schuld seines Vaters zur ,Orientierung “ - im Sinne der Bestimmung eigener Identität (durch Abgrenzung) und im Sinne einer geographisch-kulturellen Orientierung im ,Osten'.

\section{Literatur}

Agamben, Giorgio (2003): Was von Ausschwitz bleibt. Das Archiv und der Zeuge. Frankfurt (M.).

AMÉRY, JEAN (1988): Jenseits von Schuld und Sühne. Bewältigungsversuche eines Überwältigten. München.

AN-Ski (1976): Der Dibbuk. Dramatische Legende in vier Bildern. Neue deutsche Übertragung von Salcia Landmann und Horst Bienek. Mit Materialien zum Exorzismus-Thema und zur Aufführungsgeschichte. München.

Arendt, Hannah (2006 / 2008): Über das Böse. Eine Vorlesung zu Fragen der Ethik. Aus dem Nachlaß hrsg. von Jerome Kohn. Übersetzt aus dem Englischen von Ursula Ludz. Mit einem Nachwort von Franziska Augstein. München.

Assmann, Jan (2005): Das kulturelle Gedächtnis. Schrift, Erinnerung und politische Identität in frühen Hochkulturen. München.

BeiLein, Matthias (2008): 86 und die Folgen. Robert Schindel, Robert Menasse und Doron Rabinovici im literarischen Feld Österreichs. Berlin. 
Breysach, Barbara (2005): Schauplatz und Gedächtnisraum Polen. Die Vernichtung der Juden in der deutschen und polnischen Literatur. Göttingen.

Celan, Paul (1970): Ausgewählte Gedichte. Zwei Reden. Nachwort von Beda Allemann. Frankfurt (M.).

Diem, Peter (1995): Die Symbole Österreichs. Zeit und Geschichte in Zeichen. Mit über 300 Schwarzweiß- und Farbabbildungen. Salzburg.

FRANK, NiKLAS (1987): Der Vater. Eine Abrechnung. München.

FREUd, SigMUND (1989): Vorlesungen zur Einführung in die Psychoanalyse. Studienausgabe. Bd. I. Frankfurt (M.), 31-445.

- (1989): Das Unheimliche. Studienausgabe. Bd. XII. Frankfurt (M.), 227-268.

- (2000): Das Ich und das Es. Studienausgabe. Bd. III. Frankfurt (M.), 273-330.

GLATZER, NAHUM (1987): Frauen in Kafkas Leben. Zürich.

HöBel, WolfGang (1992): Wien ist kein guter Platz zum Sterben. Nach drei Gedichtbänden legt Robert Schindel seinen ersten Roman vor: „Gebürtig“. In: Süddeutsche Zeitung, 26.3.1992, Beilage:II.

JASPERS, KARL (1963): Lebensfragen der deutschen Politik. München.

KASZYŃSKI, STEFAN H. (2006): W cieniu habsburskich krajobrazów. Trzynaście esejów o literaturze austriackiej. [Im Schatten der habsburgischen Landschaften. Dreizehn Essays über die österreichische Literatur]. Poznań.

KOPACKI, ANDRZEJ (2009): Literatura samonegacji. Postawy narracyjne $w$ prozie niemieckojęzycznej przełomu XX i XXI wieku. [Literatur der Selbstleugnung. Narrative Grundlagen in der deutschsprachigen Prosa am Übergang vom 20. zum 21. Jahrhundert]. Warszawa.

LACKNER, ERnA (2004): Generation Haß. Martin Pollacks dramatische Vatersuche. In: Frankfurter Allgemeine Zeitung, 6.10.2004:L16.

LeVI, PRIMO (2009): Ist das ein Mensch? Ein autobiographischer Bericht. Aus dem Italienischen von Heinz Riedt. München.

Pollack, Martin (1994): Nach Galizien. Von Chassiden, Huzulen, Polen und Ruthenen. Eine imaginäre Reise durch die verschwundene Welt Ostgaliziens und der Bukowina. Mit 40 Abbildungen. Wien.

- (2004): Der Tote im Bunker. Bericht über meinen Vater. Wien.

- (2005): Wywiad. [Interview]. In: Gazeta Wyborza, 9.5.2005.

RABINOVICI, Doron (1997): Suche nach M. Roman in zwölf Episoden. Frankfurt (M.).

- (2001): Credo und Credit. Einmischungen. Frankfurt (M.).

Rosten, Leo (2006): Jiddisch. Eine kleine Enzyklopädie. Aktualisiert und kommentiert von Lawrence Bush. Illustriert von R. O. Blechmann. Übersetzung und deutsche Bearbeitung von Lutz-W. Wolff. München. 
Alexander Höllwerth

ROTHE, KATHARINA (2009): Das (Nicht-)Sprechen über die Judenvernichtung. Psychische Weiterwirkungen des Holocaust in mehreren Generationen nicht-jüdischer Deutscher. Gießen.

SAID, Edward W. (2004): Freud und das Nichteuropäische. Mit einer Einführung von Christopher Bollas und einer Replik von Jacqueline Rose. Deutsch von Miriam Mandelkow. Zürich.

SCHINDEL, ROBERT (1992): Gebürtig. Roman. Frankfurt (M.).

Schmidt-Dengler, Wendelin (1997): Der andere Name. In: Die Presse, 8.3.1997, Beilage:V.

Schmitz-Berning, Cornelia (2000): Vokabular des Nationalsozialismus. Berlin/New York.

STEINECKe, HARTMUT (2006): Erinnerung an die Shoa in der „Vergessensstad"“: Robert Schindel und Robert Menasse. In: DRYNDA, JoAnNA / DzIKOWSKA, KATARZYNA (eds.): Labyrinthe der Erinnerung. Beiträge zur österreichischen Literatur. Festschrift für Prof. Stefan H. Kaszyński. Poznań, 239-251.

Steinmann, Kurt (2002): Nachwort. In: SOPHOKLES: König Ödipus. Übersetzung und Nachwort von Kurt Steinmann. Stuttgart, 77-84.

Teufel, ANeTte / Schmitz, Walter (2007): Wahrheit und „subversives Gedächtnis “. Die Geschichte(n) von Vladimir Vertlib. In: VertLib, VladimiR: Spiegel im fremden Wort. Die Erfindung des Lebens als Literatur. Dresdner ChamissoPoetikvorlesungen 2006. Mit einem Nachwort von Anette Teufel und Walter Schmitz sowie einer Bibliographie. Dresden, 201-253.

Vogel, WALter (1992): „Mauthausen ist eine schöne Stadt“. Messerscharfer Witz aus dem Geiste des Galgens: Robert Schindel und sein Roman „Gebürtig“. In: Die Presse, 16.5.1992, Beilage:VII. 